\title{
Quasi-Octave Bandwidth Phase Matched K/Ka Antenna Feed Subsystem for dual RHCP/LHCP Polarization
}

\author{
Angel Mediavilla, Juan L. Cano, Karen Cepero \\ Dep. Ingeniería de Comunicaciones \\ Universidad de Cantabria \\ 39005, Santander, Spain \\ juanluis.cano@unican.es
}

\begin{abstract}
The architecture concept and fabrication of a quasioctave bandwidth waveguide feed network for circular polarized antennas is discussed here. The main advantages of this extremely compact topology rely on three key achievements: the instantaneous bandwidth capability, the axial ratio improvement and the significant size reduction which is in many cases determinant in antenna feed subsystems. Experimental measurements exhibit very good agreement with the predicted results given by conventional mode matching techniques. The overall structure provides an axial ratio as low as $0.7 \mathrm{~dB}$ along with a return loss better than $25 \mathrm{~dB}$, at every waveguide port, over the whole bandwidth $(60 \%)$, which represents the today's state-of-the-art. Moreover, yield analysis and simple scaling properties assure the use up to $Q$ band.
\end{abstract}

Keywords-Satellite Communications; Antenna feed; Turnstile Junction; ridged polarizer; mode transformer; octave bandwidth.

\section{INTRODUCTION}

Many communication waveguide feed systems use dual circular polarization to avoid the physical alignment during the installation process and to increase the capacity and versatility of the modern LOS (line-of-sight) and satellite links. For this purpose, a compact orthomode transducer (OMT)-Septum or a linear OMT followed by a polarizer is inserted between the solid state amplifier and the antenna feed horn that drives the main reflector geometry. Additional diplexers could be used to separate frequency sub-bands.

Several structures have been proposed in the literature over the last years, and a survey of waveguide circuits that realize individual polarizer and OMT functions can be found in references [1] to [6]. As an example, these polarizers are capable of operating up to $50 \%$ bandwidth, and some OMT designs can reach up to $60 \%$ bandwidth with isolations better than $55 \mathrm{~dB}$. These individual achievements are not longer valid when connecting together the subsystems in an octave bandwidth configuration, and thus there is an inherent electrical degradation mainly due to high order modes interaction along with fundamental mode standing wave appearance. To avoid these inconveniences, each feed subsystem, including the square to circular transducers, must be carefully designed in such way that not only the individual performances have to be maximized but also an strict control on the high order mode generation have to be addressed.

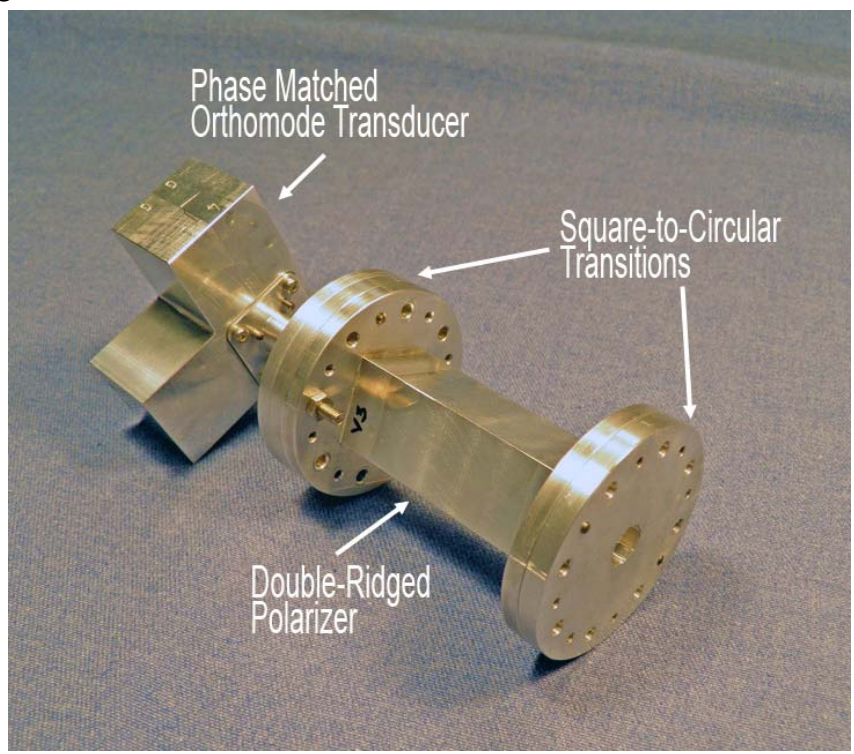

Figure 1. Complete $\mathrm{K} / \mathrm{Ka}$ band waveguide feed

Therefore, this paper presents and discusses a compact waveguide feed network, Fig.1, that overcomes previous bandwidth limitations thus approaching the theoretical limit for circular polarization. More precisely, simple scaling properties assure their use up to millimeter frequency bands.

\section{SUBSYSTEMS DESIGN}

\section{A. Orthomode Transducer}

The theoretical operating bandwidth of any orthomode transducer is constrained between the cut-off frequencies of the fundamental modes in the three physical ports and the cut-off frequency of the first higher order mode that can propagate at the access terminals. On the other hand, phase matched outputs require equal routing paths for both linear horizontal and vertical polarizations while maintaining electromagnetic symmetry. To fulfill both requirements in quasi-octave bandwidth operation, a modification of the previous works 
described in [7] and [8] was used. Turnstile-based reduced height topology, five-section circular scatter element and a combination of step transformer plus simple H-plane iris allow the circuit to operate in a $65 \%$ fractional bandwidth with return losses better than $35 \mathrm{~dB}$ at the waveguide ports.

Fig. 2 shows a picture of the proposed OMT. The structure uses a slightly modified WR34 standard rectangular waveguide and a common circular port having a diameter of $9.9 \mathrm{~mm}$. The OMT body is divided into four pieces, machined separately, which join along the circular waveguide axis and consequently split the rectangular waveguides at their E-field and $\mathrm{H}$-field mid-planes respectively.

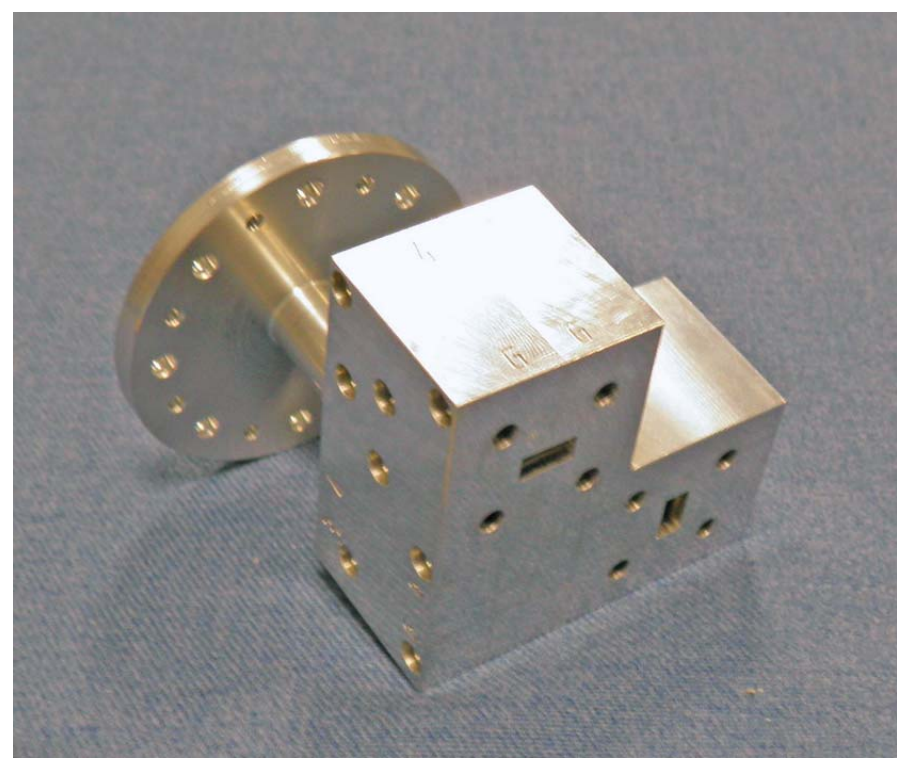

(a)

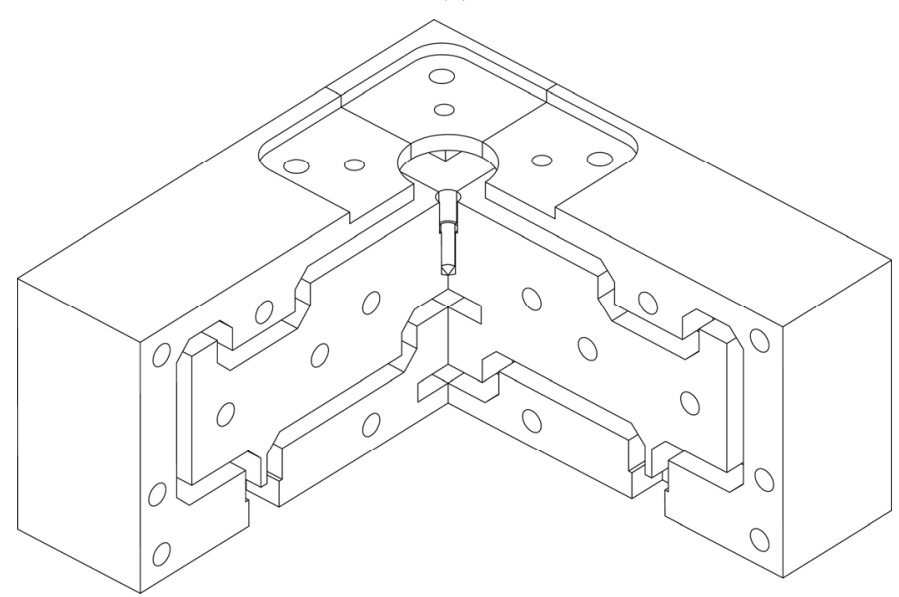

(b)

Figure 2. a) Orthomode transducer picture; b) internal view

In order to measure the whole bandwidth, two overlapping standard rectangular waveguides have been used: WR34 and WR28, and the results from both measurements are put together in the same graph. To measure at the common port, two highly efficient rectangular to circular waveguide transitions were used along with a dedicated circular waveguide TRL calkit. Fig.3 summarizes the measurement results. Experimental data shows return losses better than 35 $\mathrm{dB}$ in the $18-35 \mathrm{GHz}(64 \%)$ bandwidth while the isolation is better than $55 \mathrm{~dB}$. These results are one of the key points to assure quasi-octave bandwidth operation for the complete antenna feed. This is because in such structures the absence of spikes in the measured data means absence of any higher order mode propagation.

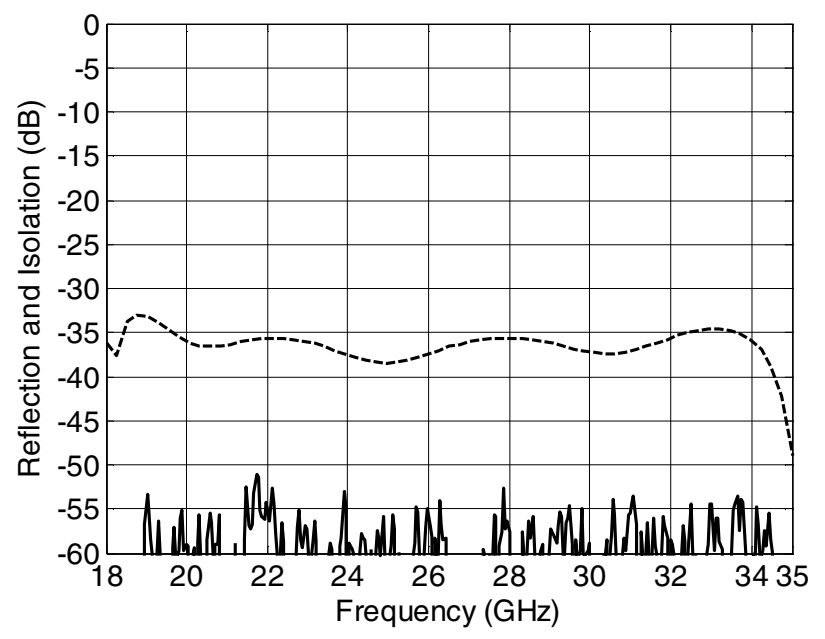

Figure 3. Orthomode transducer experimental data; reflection at the circular port in dashed line; and isolation in solid line.

\section{B. Circular Polarizer}

As stated above, some innovative polarizers using both circular or square cross section [9] are capable to provide up to $60 \%$ fractional bandwidth along with an extremely flat axial ratio $(<0.4 \mathrm{~dB})$. Although these structures exhibit very good return losses $(<24 \mathrm{~dB})$, these numbers are not enough for the purpose of a complete antenna feed network having an expected cross-polar better than $30 \mathrm{~dB}$ in the whole bandwidth.

To overcome such inconveniences, a modified version of the circuit described in [9] was used. The squared cross section was maintained, as well as the dual double-ridge approach, to guarantee $90^{\circ}$ differential phase shift for the two orthogonal modes $\left(\mathrm{TE}_{10}\right.$ and $\left.\mathrm{TE}_{01}\right)$. This is because the use of ridges reduces the cutoff frequency of the fundamental mode and increases proportionally the cutoff frequency of the higher order modes. Moreover, the two pairs of opposing ridges have a similar geometry and hence the phase difference between the two orthogonal modes varies smoothly with the frequency.

The critical improvement, Fig.4a, concerns the one-sided corrugation where, instead of using uniform gratings $g(z)=K$, we use a cosinusoidal profile given by (1):

$g(z)=g_{0}+g_{1} \cos \left(\frac{\pi \cdot z}{L_{g}}\right)$

where $L g$ is the total corrugated length, $g_{0}$ and $g_{l}$ are weighting parameters, and $z$ the longitudinal axis dimension. Other profiles such as squared cosine, as well as cubic or circumferential shapes, do not provide substantial improvement in terms of axial ratio. 
To assure symmetry, compactness and good differential phase shift, the structure was built, Fig.4b, using wire electrical discharge along with conventional milling machining processes on a single aluminum block. The final circuit is $65 \mathrm{~mm}$ long having $N=14$ corrugations and five matching steps. As in the OMT case, it was necessary to design two overlapping rectangular to square transformers along with a dedicated square waveguide thru-reflect-line (TRL) calkit.

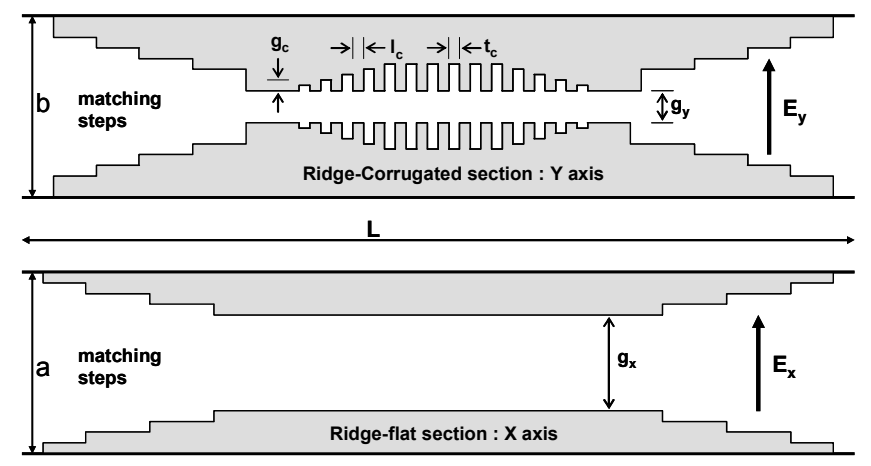

(a)

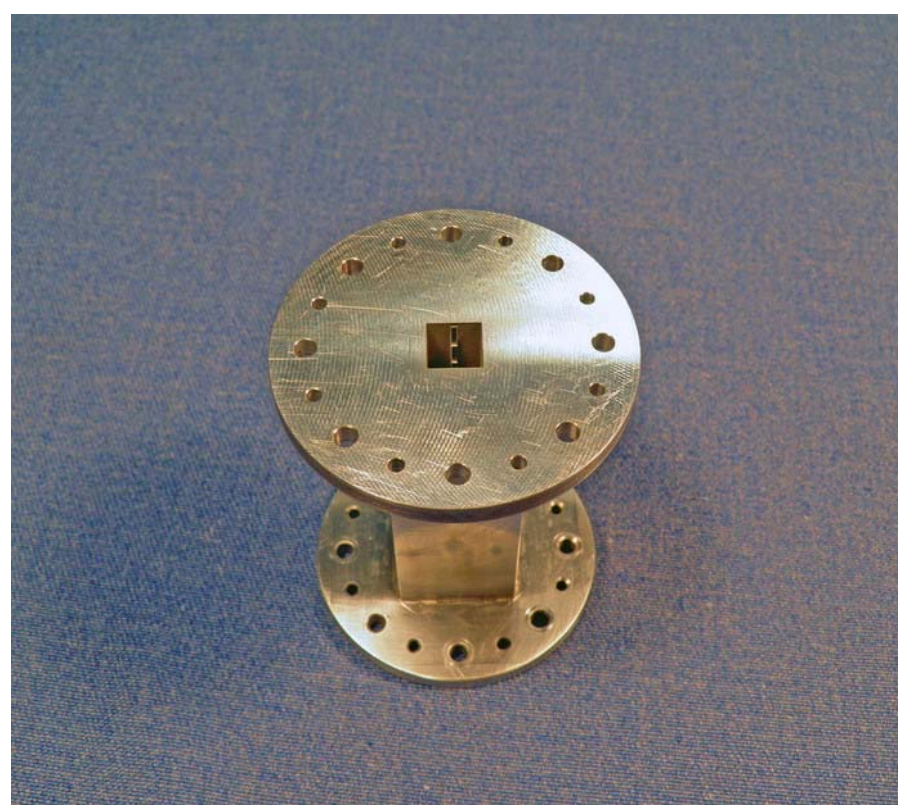

(b)

Figure 4. a) Polarizer internal view, b) Picture of the Polarizer

Fig.5 shows the measured return loss as well as the differential phase error with respect to the nominal value $90^{\circ}$. Apart from the excellent overall matching for the two orthogonal polarizations, the phase error remains in the range of $\pm 1^{\circ}$ which corresponds to an axial ratio better than $0.15 \mathrm{~dB}$. This is the second key point that will allow octave bandwidth operation for the complete system.

\section{Square to Circular Transition}

In most of cases the antenna elements use circular waveguide as driving port. On the other hand our broadband OMT uses circular cross section at the common port. These two boundary conditions obligate the use of a pair of square to circular transducers that match the square waveguide provided by the polarizer. These transducers must offer very low losses, higher order mode free operation and exceptional return losses in a broadband operation.

For this purpose, an extended version, Fig.6a, of the octagonal approach described in [10] was used to obtain a true $3-\mathrm{dB}$ power split, when connecting in a $45^{\circ}$ configuration, and return losses in the range of $40 \mathrm{~dB}$.

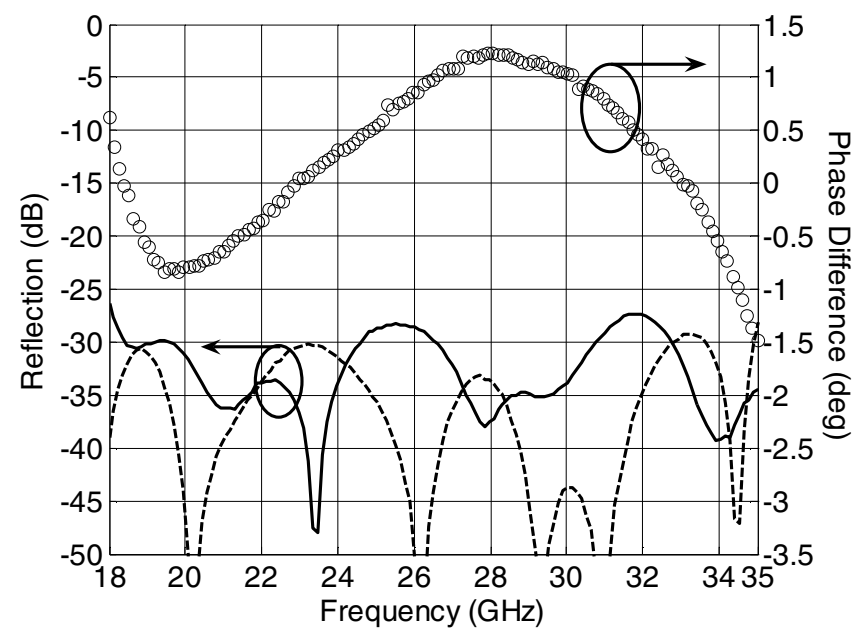

Figure 5. Polarizer experimental data; reflection of the orthogonal polarizations in the square waveguide, $\mathrm{TE}_{10}$ and $\mathrm{TE}_{01}$ (solid and dashed lines); phase difference between the orthogonal modes (circles).

This octagonal transducer uses four specific intermediate sections having almost the same cutoff wavelength as the two standard connecting waveguides. This strategy assures theoretical frequency-independent impedance transformation ratio in a very short length, which is the third key point for the complete antenna feed.

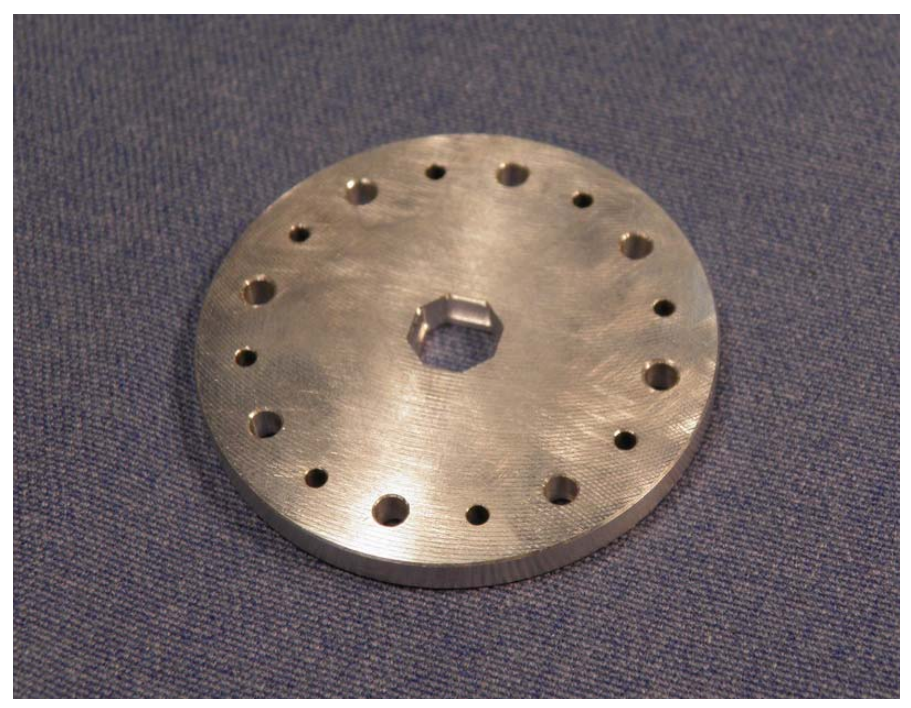

(a) 


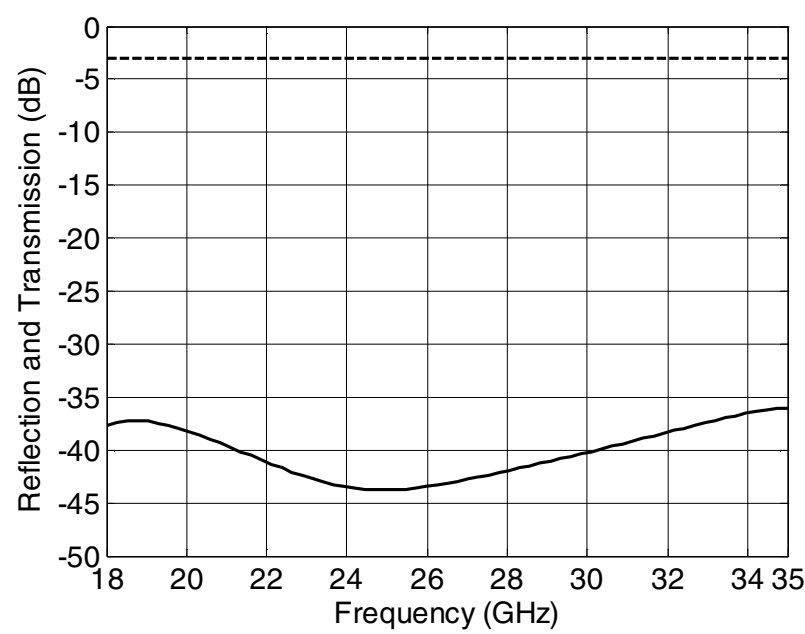

(b)

Figure 6. a) Picture of the square to circular transducer, b) Experimental results; reflection in solid line, and transmission in dashed line.

Fig.6b shows the experimental measurements for this transducer in terms of the $3 \mathrm{~dB}$ division and return loss. The total length of this element is less than $8 \mathrm{~mm}$ which is fully compatible with the shortness constraint.

\section{Complete Feed}

Fig.1 shows the complete assembly. To measure the antenna feed it was necessary to design and fabricate a circular waveguide octave-bandwidth radiating load: a single conical horn where, independently of the radiation pattern, the broadband return loss is always better than $40 \mathrm{~dB}$ to be sure that this mismatch does not degrade the feed axial ratio.

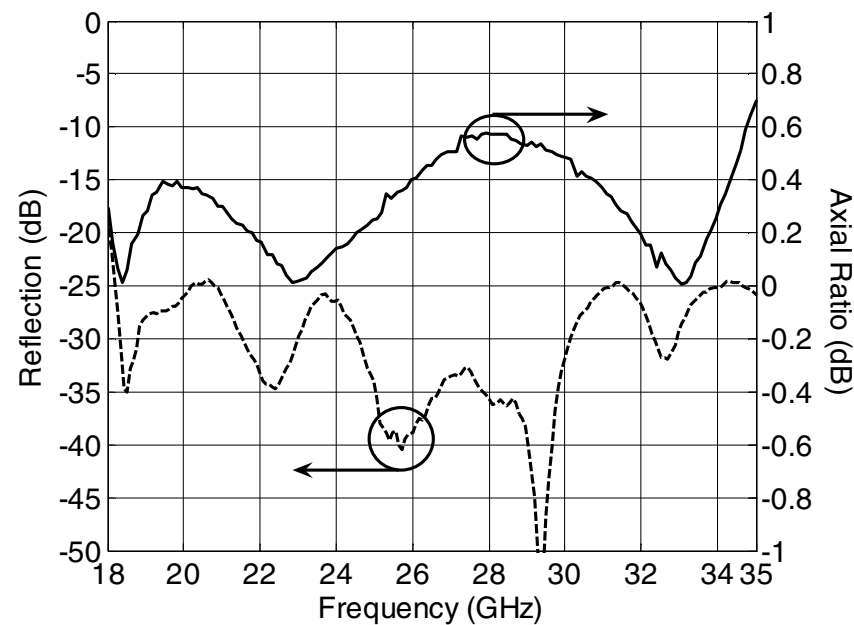

Figure 7. Antenna feed experimental results; reflection at one rectangular port is shown in dashed line; the axial ratio is plotted in solid line.

Fig.7 shows the experimental measurements for the complete feed. As expected from this step-by-step design strategy, the return losses concerning the rectangular waveguide ports remain better than $25 \mathrm{~dB}$ while the overall axial ratio is always lower than $0.7 \mathrm{~dB}$ in the whole bandwidth. Furthermore, the total length for this feed is less than $135 \mathrm{~mm}$ which constitute a strategic size reduction for antenna feed subsystems.

\section{CONCLUSIONS}

An ultra-broadband ( $>60 \%$ fractional bandwidth) waveguide feed network for circular polarized antennas has been designed and tested. The overall structure provides an axial ratio as low as $0.7 \mathrm{~dB}$ along with a return loss better than $25 \mathrm{~dB}$, at every waveguide port, over the whole bandwidth, which represents the today's state-of-the-art in terms of electrical performances. Moreover, compactness and extremely small size have been achieved through the use of accurate intermediate designs that assure excellent overall electrical behavior for the complete antenna feed. Yield analysis and simple scaling properties assure the use up to $\mathrm{Q}$ band.

\section{ACKNOWLEDGMENT}

Authors wish to thank to INDRA-SPACE for their useful suggestions and technical support.

\section{REFERENCES}

[1] A.M. Boifot, E. Lier and T. Schaug, "Simple and Broadband Orthomode Transducer”, Proc. Inst. Elect. Eng., Vol. 137, No.6, pp.396-400, June 1990.

[2] A. Navarrini, R.L. Plambeck, "A Turnstile Junction Waveguide Orthomode Transducer”, IEEE Trans. On MTT, Vol. 54, No. 1, pp. 272277, Jan. 2006.

[3] S. G. Park, H. Lee, Y. H. Kim, "A Turnstile Junction Waveguide Orthomode Transducer for the Simultaneous Dual Polarization Radar", Proc. Asia Pacific Microw. Conf. APMC'09, pp. 135-138, Singapore 2009.

[4] J. Uher, J. Bornemann, U. Rosemberg, "Waveguide Components for Antenna Feed Systems: Theory and CAD”, Boston, Artech House, 1993.

[5] E. Lier, T.S. Pettersen, "A Novel Type of Waveguide Polarizer with Large Cross-Polar Bandwidth", IEEE Trans. on MTT, Vol. 36, pp. 1531-1534, Nov. 1988

[6] P. Chambelin, J. F. Pintos, "Design and Optimization of Dual and Wide Band Polarizer for Low Cost Ka Band Application", IEEE Antennas and Propagation Society International Symp. 2006.

[7] J.L. Cano, A. Tribak, R. Hoyland, A. Mediavilla, E. Artal, "Full Band Waveguide Turnstile Junction Orthomode Transducer with Phase Matched Outputs", International Journal of RF and Microwave Computer Aided Engineering, Wiley Publishers, Vol.20, №3, pp.333341, may 2010.

[8] A. Tribak, J.L. Cano, A. Mediavilla, M. Boussouis, "Octave Bandwidth Compact Turnstile-Based Orthomode Transducer", IEEE Microwave and Wireless Components Letters, Vol. 20, No. 10, pp. 539-541, october 2010.

[9] A. Tribak, A. Mediavilla, J.L. Cano, M. Boussouis, K. Cepero, "UltraBroadband Low Axial Ratio Corrugated Quad-Ridge Polarizer", Proc of the 39th European Microwave Conference, pp, 73-76, sept. 2009, Roma.

[10] A. Mediavilla, J.L. Cano, K. Cepero, "On the Octave Bandwidth Properties of Octagonal-Shaped Waveguide Mode Transformers", IEEE Trans. on MTT, Vol. 59, № 10, pp. 2447-2451, October 2011. 